\title{
Perfil pedagógico: una cara del docente con varias aristas
}

\section{Pedagogical profile: a face of the teacher with several edges Perfil pedagógico: um rosto do professor com arestas diversas}

\author{
Gia Yerut Vilchez Malca \\ giavilchez@upeu.edu.pe \\ iD https://orcid.org/0000-0002-1619-7798 \\ Universidad Peruana Unión, Perú \\ María Nicole Dávila Vicencio \\ mariadavilav@upeu.edu.pe \\ https://orcid.org/0000-0002-0040-7105 \\ Universidad Peruana Unión, Perú \\ Jesús Marino Gomez Miguel \\ jgomezmiguel1@gmail.com \\ iD https://orcid.org/0000-0003-0706-072X \\ Universidad Nacional Daniel Alcides Carrión
}

\author{
Elmer Jared Reque Campos \\ elmerrequec@upeu.edu.pe \\ iD https://orcid.org/0000-0001-6446-6270 \\ Universidad Peruana Unión, Perú \\ Hilser Juan Castillo Paredes \\ hitlser@gmail.com \\ https://orcid.org/0000-0002-7231-774X \\ Universidad Nacional Daniel Alcides Carrión \\ Moisés Agustín Cristóbal \\ jomoelca@gmail.com \\ iD https://orcid.org/0000-0003-3828-5665 \\ Universidad Nacional Daniel Alcides Carrión
}

Recibido: 03 de enero de 2021

Aceptado: 06 de junio de 2021

\section{Resumen}

Para propios y extraños de la educación, es de mucho interés el abordaje del perfil pedagógico del docente, entendiendo que este perfil equivale solamente a una cara del docente, cuyas aristas son varias y complejas. El objetivo de este artículo es determinar las dimensiones y sus respectivos componentes del perfil pedagógico del docente, abordando de este perfil sus concepciones conceptuales y las respectivas dimensiones. A final de estas consultas teóricas, se concluye que las dimensiones más saltantes del perfil pedagógicos del docente son: la capacidad de planificación y organización del plan de clase, la capacidad de planificación didáctico-metodológica, la capacidad de evaluación, cuyas dimensiones también incluyen sus respectivos componentes.

Palabras clave: perfil pedagógico, docente, planificación, organización, metodología, evaluación. 


\begin{abstract}
For both locals and foreigners of education, the approach to the pedagogical profile of the teacher is of great interest, understanding that this profile is only equivalent to one face of the teacher, whose edges are various and complex. The objective of this article is to determine the dimensions and their respective components of the pedagogical profile of the teacher, addressing from this profile their conceptual conceptions and the respective dimensions. At the end of these theoretical consultations, it is concluded that the most salient dimensions of the pedagogical profile of the teacher are: the capacity for planning and organization of the class plan, the capacity for didactic-methodological planning, the capacity for evaluation, whose dimensions also include their respective components.
\end{abstract}

Keywords: pedagogical profile, teacher, planning, organization, methodology, evaluation.

\title{
Resumo
}

Tanto para os locais como para os estrangeiros da educação, a abordagem ao perfil pedagógico do professor é de grande interesse, entendendo que este perfil equivale apenas a uma face do professor, cujas arestas são diversas e complexas. O objetivo deste artigo é determinar as dimensões e seus respectivos componentes do perfil pedagógico do professor, abordando a partir desse perfil suas concepções conceituais e as respectivas dimensões. Ao final dessas consultas teóricas, conclui-se que as dimensões mais salientes do perfil pedagógico do professor são: a capacidade de planejamento e organização do plano de aula, a capacidade de planejamento didático-metodológico, a capacidade de avaliação, cuja as dimensões também incluem seus respectivos componentes.

Palavras-chave: perfil pedagógico, professor, planejamento, organização, metodologia, avaliação.

\section{Introducción}

Abordar la educación implica ingresar en un ámbito totalmente complejo, mucho más cuando se habla de los sujetos y protagonistas de la misma, quienes son totalmente complejos en su actuación. La calidad del docente es percibida directamente por los estudiantes, quienes observan en detalle las respectivas características, las cuales les facultan la determinación de una calificación en los términos de un docente regular, bueno, docente, excelente; los diversos, escasos, múltiples y vastos conocimientos sobre 
la pedagogía son trasversales; en este contexto, el docente transfiere conocimiento, también crea y otorga muchas posibilidades para que el estudiantes realice su propia construcción (Orellana, Merellano y Almonacid, 2018). No solamente se busca la calidad del docente, también se habla mucho de la misma, ¿qué hacer para lograrla? En este sentido, se requiere ejercer mucho cuidado y pericia sobre la formación inicial de los docentes, cuya formación es sustantiva y determinante, para lo cual se requiere, en los sistemas educativos, trabajar mejorando sus competencias, sus destrezas, sus motivaciones profesionales y no profesionales, cuya situación no solamente se hace compleja sino también exigente (Muñoz, Rodríguez y Luque, 2019). En el escenario complejo de la educación, el docente es un protagonista inevitable, oficial, formal, regulador, objetivo y visible. Todo docente posee requisitos para ejercer su profesión (Chasi, 2020); por ejemplo, el perfil pedagógico o didáctico (Quijije, 2021; González, Palomares, López y Gento, 2019), el cual le habilita y le da las competencias para la planificación y organización del plan de clase, la planificación y diseño del plan de clase, el planteamiento de objetivos, la selección de contenidos, la planificación didácticometodológica, la respectiva organización de los contenidos, el uso eficiente de metodologías, la mejorada capacidad de relación teoría-práctica, la entrega de explicaciones comprensibles, ejemplos y demostraciones, la aplicación del conocimiento en situaciones reales, el uso correcto y efectivo de la TIC (Fernández y Rodríguez, 2017; Borgobello, Madolesi, Espinosa y Sartori, 2019); inclusive las competencia de evaluación docente y estudiantil. En el sentido de la evaluación, el docente debe poseer capacidades de autoevaluación, coevaluación, hetero evaluación, evaluación diagnóstica, evaluación formativa y evaluación sumativa (Acosta y Acosta, 2016).

En el escenario educativo, muchas veces se conjugan algunos conceptos en forma imprescindible; por ejemplo, práctica, teoría, docentes, procesos de aprendizaje, perfil profesional, entre otros. En realidad, la presencia de estos elementos conceptuales construye un universo complejo, el cual incide definitivamente sobre los aprendizajes de los estudiantes. El perfil del docente tiene sus repercusiones sobre la gestión, la administración y el compromiso con las acciones metodológicas y técnicas, orientadas hacia el cultivo de la creatividad y la participación de los estudiantes (Chasi, 2020).

Un buen docente es responsable, paciente, entusiasta para desarrollar su trabajo docente y pedagógico. Su interés y motivación persiguen la ampliación de su formación, cuyas perspectivas llegan hasta sus discípulos, a quienes las entrega, las comparte con 
ellos, quienes así despiertan sus propias motivaciones y sus conexiones con la realidad que les circunda y con la visión de un futuro mejor y cercano.

En esta perspectiva, se aborda el perfil pedagógico del docente, con el objetivo de determinar las dimensiones del mismo, en un espacio educativo caracterizado por su complejidad, descuidado, marginado; incluso en el cual se ha perdido las motivaciones profesionales, educativas, éticas, políticas, económicas, sociales y culturales.

\section{Perfil pedagógico del docente}

\section{Pedagogía, perfil y docente: aproximaciones conceptuales}

Para Petrus (1997, citado por Vives, 2016), la pedagogía es ciencia, gracias a la cual se construye el ámbito teórico de la educación y su respectiva disciplina. Por su parte, Vives (2016) afirma que "la pedagogía es la ciencia que estudia todos los procesos que implican la enseñanza como el aprendizaje de los sujetos en sus particularidades y contextos diferenciales" (p. 41). Definitivamente en estos conceptos quedan subrayados los conceptos sustantivos: ciencia, proceso enseñanza aprendizaje.

La pedagogía revela las competencias del docente, "para desarrollar el proceso de enseñanza-aprendizaje tanto individual como grupal", cuyas acciones se desarrollan en un ambiente de autonomía y crítica, con el uso de la tecnología y las metodologías requeridas (Espín y Alpízar, 2020).

Para Chasi (2020), el perfil “es una condición necesaria”, cuya condición posibilita al docente ejercer la orientación de los estudiantes, mediante conocimientos y actividades, en el universo del campo educativo. El perfil del docente se dirige hacia el desarrollo de las acciones de la docencia, generando motivación, búsqueda de metodologías, la creatividad, la innovación, la toma de conciencia, la participación, la búsqueda de soluciones, la búsqueda de futuro. Implica conocimientos, competencias, habilidades, cualidades, actitudes, condiciones humanas y sociales, conjunto de rasgos, profesional experto, desempeño específico, experticia, especialización, formación acreditada. Según Espín y Alpízar (2020), el perfil del docente constituye parte importante de las competencias profesionales que revelan entrenamiento, capacitación y evaluación.

El protagonista de la educación sistematizada recibe los nombres: pedagogo, maestro, profesor, docente, cuyas designaciones tienen sus connotaciones históricas, educativas, sociales y culturales. Más allá de la construcción del perfil y sus rasgos psicológicos (Vásquez et al., 2020), se pretende generar la construcción del perfil 
pedagógico del docente, se requiere construir un marco conceptual que permita encuadrar las opiniones, los comentarios, las descripciones y los análisis respectivos. En este sentido, se requiere abordar y conceptualizar la pedagogía, el maestro y su respectivo perfil pedagógico.

Flores et al. (2021) hablan de perfil de egreso de la carrera de pedagogía, con el sentido de declaración institucional sobre la formación profesional, cuyo contexto es social, quedando involucrado el profesional, sus habilidades y competencias frecuentes relacionadas con la disciplina estudiada. Este perfil permite la movilidad de los estudiantes en los espacios educativos; posee sus propios indicadores, le corresponde a cada carrera, corresponden a los planes de estudio y a los respectivos currículos profesionales y de las carreras.

En este contexto, se habla de competencias pedagógicas, para "guiar el entrenamiento/capacitación y evaluación del profesorado" (Villarroel y Bruna, 2017, p. 76). Para Ortega (2010, citado Villarroel y Bruna, 2017), el perfil del docente tiene ocho competencias:

a) competencias cognitivas (el conocimiento en un nivel disciplinar y pedagógico), b) competencias metacognitivas (pensamiento crítico, autocrítico y reflexivo), c) competencias culturales y contextuales (conocimientos del entorno y de la organización), d) competencias comunicativas (lenguaje científico verbal y escrito), e) competencias sociales (cooperación, empatía y trabajo en equipo), f) competencias de gestión (gestión, organización y planificación), g) habilidades tecnológicas (aprendizaje, investigación y uso de las TIC), h) competencias de investigación (búsqueda de información sobre metodologías y recursos) (p.77).

\section{Dimensiones del perfil pedagógico}

Competencia de planificación, diseño y organización del plan de clase

Este perfil está encaminado hacia la gestión pedagógica, en cuyo ámbito se encuentran: planificación y diseño del curso, planteamiento de objetivos y selección de contenidos (Villarroel y Bruna, 2017). Comprende todas las actividades encaminadas hacia la planificación, la ejecución, el control y la evaluación del proceso de enseñanza aprendizaje.

La planificación de la clase es indispensable y fundamental para el docente, permitiéndole el cumplimiento de sus funciones de facilitador y mediador del proceso educativo. Esta planificación se convierte en una acción de primera índole para la orientación, ejecución y control del proceso de la enseñanza. Reyes (2017) afirma que la planeación de clase es el hilo conductor de las múltiples estrategias y acciones desarrolladas dentro y fuera del aula escolar; a su vez, el plan de clase es el eje transversal, 
garantiza al docente aterrizar las actividades de manera sistémica y sistemática. Al respecto, Marín et al. (2018) declaran:

El proceso de planificación de la clase debe constituir una actividad medular, para todo docente en ejercicio, a partir de su rol como mediador y facilitador del proceso pedagógico. Pues esta actividad constituye la célula básica de cuanta actividad se programe tanto dentro como fuera del aula, ya que es precisamente durante la planeación de clases, donde podemos ver con claridad lo que queremos, cómo lo queremos, con qué vamos a lograr lo que queremos, cuándo lo lograremos y con qué instrumentos se va a medir el proceso para saber los resultados del trabajo. De ahí su carácter creativo, transformador, sistémico y sistemático (p. 90)

La planificación de clase se realiza sobre la base de las necesidades, intereses e inquietudes del alumno o estudiante, precisando lo que dirá y hará, con el propósito de generar la mediación progresiva durante el proceso, el desarrollo intelectual, la manera de pensar (Reyes, 2017).

Para Zilberstein (2016, citado por Reyes, 2017), antes de planear se debe tener en cuenta los siguientes elementos: dominar el currículo, el diagnóstico integral de los estudiantes, el contenido de la asignatura, los métodos efectivos de enseñanza, las formas de organización y los medios de enseñanza, la bibliografía básica.

\section{Planteamiento de objetivos}

En el ámbito pedagógico, un objetivo es un parámetro de evaluación a nivel de educación. En el campo de la educación, un objetivo es el resultado que se espera logre el alumno al finalizar un determinado proceso de aprendizaje. Para Tao (2016), los objetivos son pieza fundamental durante todo el proceso educativo, ejerciendo gran influencia y siendo de vital importancia al momento de organizar y conducir los contenidos, además de ser guías para determinar qué enseñar y cómo enseñarlo; por otro lado, los objetivos le permiten al docente determinar cuál ha sido el progreso del alumno, facilitando la labor al docente durante el momento de determinar cuáles aspectos deben de ser reforzados.

Tao (2016) afirma que los objetivos se clasificados, por su naturaleza, en generales y específicos; por su alcance del tiempo, en estratégicos (a largo plazo), tácticos (a mediano plazo) y operacionales (a corto plazo). Los primeros describen la finalidad; los segundos son derivados de los primeros, expresados en cantidad y tiempo. Los objetivos a largo plazo describen la labor educativa, desde inicio hasta el final o término (del inicio hasta el final del año); los tácticos o a mediano plazo comprenden los trabajos por bimestres académicos; los a corto plazo u operacionales comprenden el trabajo de un mes. Por otro lado, los objetivos ofrecen las características: medibles, claros, alcanzables, desafiantes, realistas. 
Selección y organización de contenidos

El docente selecciona los contenidos para el estudiante, quien logrará el objetivo determinado. Según Zapata (2016), los contenidos responden a las necesidades específicas y son adaptados a un contexto específico, adecuados a la realidad y situación del estudiante, con el propósito de resolver los problemas de su entorno, sin perder de vista el factor sociocultural.

Uso de metodologías

\section{Metodología del aprendizaje cooperativo: "Todos para uno y Uno para todos"}

El aprendizaje cooperativo es una metodología, cuyos maestros lo usan para agrupar a los estudiantes durante el aprendizaje de una manera positiva. Es conocido con la denominación de trabajos grupales, equipos de trabajo. Esta metodología se sustenta con la teoría relacionada con el trabajo en equipo, generando atención, implicación y adquisición de conocimientos. La principal característica es la estructura: formación de grupos integrados entre 3 y 7 personas; cada miembro cumple un rol determinado para lograr objetivos, mediante la interacción y el trabajo en forma coordinada. El aprendizaje cooperativo tiene objetivo común. Ayuda el desarrollo social, por la forma adecuada y colaborativa durante la realización de los trabajos. Permite la ayuda y la resolución de situaciones personales y grupales, en un ambiente de diversidad y complejidad (Sánchez, López y Alfonso, 2018).

\section{Metodología lúdica}

El método lúdico es un conjunto de estrategias, diseñadas con el propósito de generar un ambiente de armonía y socialización. La aplicación de actividades lúdicas en la enseñanza es una forma práctica, para generar conocimiento y mensajes curriculares, fácil, amena y divertidamente, despertando el interés de los estudiantes mediante la tecnología con juegos virtuales, practicados durante el proceso de aprendizaje de las matemáticas.

Aristizábal, Colorado y Gutiérrez (2016) afirman que la educación requiere capacidades: crítica, analítica, reflexiva. El desarrollo intelectual alto permite interpretar, argumentar, proponer, plantear y resolver problemas. La adquisición del sentido numérico, por ejemplo, se logra mediante el juego, en situaciones ricas, variadas y significativas, cuyo juego estimula inteligencia e imaginación de los estudiantes.

Metodología basada en problemas 
Esta metodología permite el desarrollo de competencias científicas (Aguado y Campo, 2018). El aprendizaje basado en problemas (ABP) mejora las habilidades de los estudiantes, para la toma de decisiones en un contexto educativo, en correspondencia al constructivismo, el trabajo colaborativo, autodirigido y contextual; es decir, protagoniza en el contexto de "la necesidad de un enfoque de aprendizaje activo, cooperativo, centrado en el estudiante" (Pérez, 2018, p. 156). Por otro lado, esta metodología desarrolla la inteligencia emocional de los estudiantes, beneficiando la educación integral y el desarrollo de las capacidades; es una metodología activa, centrada en el estudiante, cuyos fundamentos son: el argumento teórico y el argumento conceptual (Luy, 2019). El ABP estimula los aprendizajes y sus habilidades para la solución de problemas y la adquisición de conocimiento específico (Gil, 2018).

\section{Metodología basada en proyectos}

El Aprendizaje Basado en Proyectos (ABP) es una de las metodologías más utilizadas en clase actualmente. El ABP permite que los estudiantes adquieran conocimientos y competencias, mediante la solución de problemas y la elaboración de proyectos que responden a los problemas de la vida real. Se parten de un problema concreto y real, en vez del modelo teórico y abstracto tradicional; se generan evidentes mejoras en la capacidad de conocimiento; facilitan el desarrollo de competencias complejas: pensamiento crítico, comunicación, colaboración, resolución de problemas. Comprende dos metodologías: el aprendizaje basado en problemas (ABP) y el aprendizaje basado en proyectos (García y Pérez, 2018). Esta metodología permite que el estudiante realice planificación, implementación y evaluación de proyectos, con aplicaciones reales y objetivas, cuyas interacciones de los docentes y los estudiantes son óptimas (Toledo y Sánchez, 2018). El ABP se complementa con la metodología y estrategias de la investigación acción, mediante la labor docente, constituyéndose en metodologías flexibles, cuyo papel es activo y reflexivo durante todo el proceso desarrollado en el aula (Botella y Ramos, 2019). Es una metodología didáctica privilegiada, presenta diversos enfoques, genera un aprendizaje relevante, genera experiencia y conocimiento (Doménech et al., 2019).

\section{Metodología Flipped Classroom (Aula invertida)}

El método flipped classroom, denominado aula invertida; es una metodología moderna, ha ganado más popularidad durante los últimos años. Tiene su base en la TICs, permite el aprendizaje activo de los estudiantes, con el propósito de comprobar su efecto 
y beneficios para el aprendizaje y rendimiento académico. Es un modelo pedagógico, cuyos elementos tradicionales de la lección impartida por el profesor se invierten: los materiales educativos primarios son estudiados por los alumnos en casa y, luego, se trabajan en el aula (Hinojo et al., 2019). Constituye una propuesta metodológica para lograr aprendizajes en matemática (Guerrero et al., 2017). Mediante el aula invertida, la enseñanza y los procesos de aprendizaje son auto regulados y activos en las aulas, cuyo uso de recursos es autónomo, aprovechando el tiempo en forma activa por parte de los estudiantes, bajo un contexto de trabajo colaborativo y la resolución de diversos problemas (Reyes et al., 2018). Actualmente, las metodologías de aula invertida involucran la participación de los padres de familia y los estudiantes; esta metodología también es denominada clase invertida, modelo invertido de aprendizaje, cuyo rol de estudiante es más activo y participativo (Falcón et al., 2021). El modelo de aula invertida siempre promueve aprendizajes significativos, mejora la calidad y los estándares académicos, es un modelo de aprendizaje híbrido, integra entornos virtuales y entornos presenciales, con autonomía, responsabilidad, actividades complejas, trabajo cooperativo, sin dejar al margen la retroalimentación (Bohórquez y Pérez, 2021).

Capacidad de relación teoría-práctica

El docente revela práctica y las reflexione teórica, cuya capacidad es muy importante, en contexto diversos científicos, académicos, pedagógicos, sociales (Ferreiro y Domínguez, 2020). En el universo de la educación, la teoría y la práctica constituyen dos realidades autónomas; sin embargo, caminan juntas siempre. Son desarrolladas en simultáneo, ambas constituyen un eterno problema para los educadores, los filósofos y los políticos. Así han surgido dos enfoques: el enfoque científico-tecnológico y el enfoque hermenéutico-interpretativo, la supremacía de la teoría sobre la práctica, para el primer enfoque; para el segundo, la supremacía de la práctica. En el marco de las metodologías participativas se circunscribe la participación teórico práctica (Suárez, 2021). Se enmarca en el plano de las conjeturas y las demostraciones en el aula (Balaguera, 2020).

Entrega explicaciones comprensibles, ejemplos y demostraciones

El docente es muy claro entregando los mensajes a sus estudiantes; pues les entrega mensajes comprensibles; también conoce perfectamente bien a su audiencia (diversos niveles académicos, sociales y culturales). La información que les entrega es coherente y clara; utiliza diversas herramientas (ejemplos, cuentos, anécdotas); genera interés y empatía. Genera creencia, confianza, seguridad en la mente de los estudiantes, al 
entregarles conocimiento e información. El perfil del docente implica roles, procesos, propuestas, eficacia, eficiencia, logros educativos (Fernandez, 2016).

\section{Aplicación del conocimiento en situaciones reales.}

Para Dávila et al. (2013) el estudiante tiene el deber de aprender lo que declara el docente, quien conoce exclusivamente qué enseña y cómo enseña; significa que el docente es práctico, objetivo y real. Significa también compartir experiencias de la vida real. Los docentes responden a los problemas de la vida real. El perfil pedagógico responde a la aplicación de conocimientos a situaciones reales. Los problemas en el ámbito de la educación son múltiples, diversos; desgraciadamente, la educación no es para todos, existen brechas cognitivas y digitales; la diferencia educativa aparece en los diversos estratos sociales, cuya solución es demandada a los docentes. Según Rodríguez y Alamilla (2018), los conocimientos y los saberes de los docentes, relacionados con la práctica, son complejos, por su contenido y representación. Se ha discutido, se discute y se discutirá mucho sobre la relación de la teoría y la práctica, fuentes del saber docente; ambas tienes divorcio, cuya relevancia para unos está en la teoría; para otros, en la práctica.

Significa que los docentes consideran el tiempo, los espacios y las circunstancias, los cuales demanda la aplicación del conocimiento acomodado a estas circunstancias; por ejemplo, acomodado a la virtualidad generada por la COVID 19, pasando por situaciones complejas e inciertas, en el contexto social, educativo, familiar, económico, político; significa la reconstrucción del conocimiento práctico, real, objetivo (Soto et al., 2021). Uso de las TICs

El conocimiento tecnológico es fundamental, consolida las competencias, afianza la educación, la calidad, la formación integral. Gracias al conocimiento de la TICs, los docentes replantean las actividades tradicionales de enseñanza, las amplían y complementan con nuevas actividades y recursos de aprendizaje, respondiendo a los cambios y las innovaciones de la sociedad. Las TICs han transformado el proceso educativo; han modificado la forma como docentes y estudiantes acceden al conocimiento y la información. El educador construye espacios virtuales para la educación y el intercambio de contenido científico. Las TICs constituyen una herramienta muy valiosa, desarrollan la capacidad de acceso al conocimiento; facilitan participación y realización de tareas en respuesta a las necesidades e intereses de los estudiantes (Fernández y 
Rodríguez, 2017). Se habla de la sociedad del conocimiento, mucho más en el espacio de los jóvenes; se habla de la "generación muda", sus textos son escritos en las redes sociales; su crecimiento es exponencial, en los centros y en los entornos de formación, aprendizaje, cuyos docentes administran y gestionan herramientas e instrumentos, destinados a la remisión de información y la creación de entornos, donde se practique el trabajo colaborativo (Marín y Cabero, 2019).

\section{Competencia de evaluación docente y estudiantil}

En este caso, la evaluación se constituye en método de observación del avance académico, en los dos espacios: uno del docente y otro del estudiante. La finalidad de la evaluación docente es fortalecer la profesión, permite reflexionar sobre el desempeño docente, para lograr mejores resultados en la parte de los estudiantes. Permite determinar las fortalezas y las debilidades relacionadas con el proceso enseñanza-aprendizaje. Gracias a la evaluación, los estudiantes potencian su comprensión, análisis, interpretación. Comprende varios tipos de evaluación: autoevaluación, coevaluación, hetero-evaluación (en el docente y el estudiante); por las funciones de evaluación: diagnóstica, formativa y sumativa (Arreola, 2019).

\section{Autoevaluación}

Es la evaluación personal y crítica del docente y del estudiante, con el propósito de determinar las debilidades y las fortalezas. Calatayud (2018) afirma que la formación en autoevaluación beneficia el desarrollo y el crecimiento personal y profesional.

Por su parte, Martínez et al. (2018) aprecian que la autoevaluación y reflexión favorecen y construyen caminos hacia el desarrollo profesional. Es tipo de evaluación permite la reflexión del docente y del estudiante sobre su comprensión, estrategias, metodología, factores intervinientes, comodidad, rutina, alteraciones, dinámicas de trabajo y aprendizaje, comunicación, individualismo, enseñanza, aprendizaje, saberes, habilidades, dentro y fuera del ámbito educacional.

\section{Coevaluación}

Este tipo de evaluación se realiza con la participación de docentes y estudiantes, de uno a otro y viceversa; esta evaluación es recíproca (persona o equipo), quienes poseen la misma responsabilidad, compartida durante el proceso de evaluación; en este contexto, el diálogo es imprescindible, también los consensos, las valoraciones. Fomenta la comunicación, la confianza, la empatía, flexibilidad, sensibilidad de los docentes con los estudiantes y viceversa. Mejora el ambiente de trabajo (Gómez y Quesada, 2017). 


\section{Hetero evaluación}

La hetero evaluación la realiza una persona a otra sobre su trabajo, rendimiento, desempeño, motivación, entre otros; esta evaluación se diferencia de la coevaluación, porque esta se realiza entre pares; en cambio, la hetero evaluación se realiza hacia otra persona; por ejemplo, de un estudiante a otro, a su docente; también de un docente a otro. Consiste en la valoración de las competencias y los rendimientos logrados (Cardona, Jaramillo y Navarro, 2016).

También comprende la evaluación del estudiante al docente, en las universidades, de acuerdo con los criterios establecidos, sobre el material utilizado, las evaluaciones, el proceso de enseñanza, el ambiente del aula, dominio de contenidos, entre otros (Vera, Bueno, Calderón y Medina, 2018).

\section{Evaluación diagnóstica}

Las funciones determinan el momento cuando se da evaluación de la enseñanza y del aprendizaje, por lo que se divide en tres: diagnóstica, formativa y sumativa (Segura, 2018). La evaluación diagnóstica se realiza al inicio de una actividad académica, del ciclo escolar, del semestre universitario, cuyo propósito es determinar el nivel del conocimiento, la habilidad, la actitud del estudiante; la información preliminar le sirve al docente, para realizar adecuaciones de los contenidos y la implementación de actividades académicas programadas (Sánchez, 2018)

\section{Evaluación formativa}

La evaluación formativa se realiza para monitorear el progreso del aprendizaje, para proporcionar realimentación al estudiante; se realiza sobre sus logros, deficiencias, generando oportunidades para ejercer la mejora. Se recaba información sobre el proceso enseñanza-aprendizaje, para que los docentes tomen decisiones sobre qué y cómo enseñan y aprenden los alumnos, generando mejor desempeño y motivación. Muestra las fallas, los desaciertos, las flaquezas, también las fortalezas de los docentes y los estudiantes. Tiene fines y motivaciones correctivas, reflexivas (Sánchez, 2018).

Segura (2018) afirma que esta evaluación genera apoyos pedagógicos, refuerza los procesos de aprendizaje y de evaluación; permite el uso de estrategias y técnicas diversas, de acuerdo con los estilos y ritmos de aprendizaje; facilita el análisis de los contextos, la procedencia de los estudiantes, la condición socioeconómica de los estudiantes, la presencia de barreras. Involucra los demás tipos de evaluación. Genera correctivos diversos, para mejorar el proceso enseñanza-aprendizaje. 
Se estimulan los logros de los estudiantes, reforzando las conductas, en forma positiva, evitando la censura, evitando el llamado de atención en forma negativa (Sánchez, 2018). La evaluación formativa responde a los aprendizajes por medio del error, generando un proceso de reflexión al momento de la corrección acertada, dejando el nuevo conocimiento; la retroalimentación tiene una función formativa, orienta el aprendizaje (Canabal y Margalef, 2017).

\section{Evaluación sumativa}

Esta evaluación equivale a la suma de valoraciones cuantificadas durante el proceso enseñanza aprendizaje, con el propósito de validar el logro de los objetivos, otorgando calificaciones cuantitativas, se acuerdo con el sistema de evaluación establecido. Involucra los exámenes realizados de acuerdo con la programación, en periodos y unidades determinados. Las calificaciones cuantitativas conducen a la generación de promedios, la calificación o nota final. Esta evaluación sirve para la promoción de los estudiantes, así son promovidos de ciclo, de grado, etc. (Sánchez, 2018).

\section{Conclusiones}

En este trabajo se ha intentado construir un abordaje teórico sobre las diversas dimensiones del perfil docente, con el sentido de conocer y analizar algunas de las múltiples y complejas caras del docente, reveladas durante el ejercicio de su profesión y durante la realización del proceso enseñanza aprendizaje. En este sentido, se concluye que el perfil pedagógico del docente, según el presente estudio, es totalmente complejo; en este estudio, por ejemplo, se presentan las dimensiones: competencia de planificación y organización del plan de clase, competencia de planificación didáctico-metodológica, competencia de evaluación docente y estudiantil.

Así mismo, se entiende que la competencia de planificación y organización del plan de clase, comprende la planificación y diseño del plan de clase, el planteamiento de objetivos y la selección de contenidos. Por su parte, la competencia de planificación didáctico-metodológica incluye la organización de los contenidos, el uso de metodologías, la capacidad de relación teoría-práctica, la entrega explicaciones comprensibles, ejemplos y demostraciones, la aplicación del conocimiento en situaciones reales y el uso de la TIC. No ha quedado al margen la competencia de evaluación docente y estudiantil, cuyos componentes son: la autoevaluación, la coevaluación, la hetero evaluación, la evaluación diagnóstica, la evaluación formativa y la evaluación sumativa. 


\section{Referencias}

Acosta, B. y Acosta, M. (2016). Modelos de evaluación para la acreditación de carreras. RMIE Revista Mexicana de Investigación Educativa, 21(71), 1249-1274.

https://revistas.usantotomas.edu.co/index.php/riiep/article/view/6416/6058

Aguado, A. M. y Campo, Á. A. (2018). Desarrollo de competencias científicas en Biología con la metodología del Aprendizaje Basado en problemas en estudiantes de noveno grado. Bio-Grafía, 11(20), 67-78.

https://revistas.pedagogica.edu.co/index.php/bio-grafia/article/view/8594/6511

Aristizábal, J. H., Colorado, H. y Gutiérrez, H. (2016). El juego como una estrategia didáctica para desarrollar el pensamiento numérico en las cuatro operaciones básicas. Sophia, 12(1), 117-125. www.scielo.org.co/pdf/sph/v12n1/v12n1a08.pdf

Arreola, R. L. (2019). Formación y evaluación docente basada en un perfil por competencias. Una propuesta desde la práctica reflexiva. Revista Educación, 43(2), 1-19. https://doi.org/10.15517/revedu.v43i2.30898

Balaguera, E. (2020). Conjetura y demostración en el aula en la formación de docentes. Revista Interamericana de Investigación, Educación y Pedagogía, 14(1), 177-205. https://doi.org/10.15332/s1657-107X

Bohórquez, Y. A. y Pérez, J. (2021). Aula invertida como estrategia para promover aprendizajes significativos en matemáticas en estudiantes de básica secundaria [Tesis, Universidad de la Costa]. https://repositorio.cuc.edu.co/handle/11323/8020

Borgobello, A., Madolesi, M., Espinosa, A. y Sartori, M. (2019). Uso de TIC en prácticas pedagógicas de docentes de la Facultad de Psicología de una universidad pública argentina. Revista de Psicología, 37(1), 279-317.

https://doi.org/10.18800/psico.201901.010

Botella, A. M. y Ramos, P. (2019). Investigación-acción y aprendizaje basado en proyectos: Una revisión bibliográfica. Perfiles Educativos, XLI(163), 127-141. http://www.scielo.org.mx/pdf/peredu/v41n163/0185-2698-peredu-41-163-127.pdf

Calatayud, A. (2018). La autoevaluación. Una propuesta formativa e innovadora The self-evaluation. A formative and innovative proposal. Revista Iberoamericana de Educación, 76(2), 135-152. https://doi.org/10.35362/rie7623081 
Canabal, C. y Margalef, L. (2017). La retroalimentación: La clave para una evaluación orientada al aprendizaje. Profesorado. Revista de Currículum y Formación de Profesorado, 21(2), 149-170. https://www.redalyc.org/pdf/567/56752038009.pdf

Cardona, S. A., Jaramillo, S. y Navarro, Y. (2016). Evaluación de competencias con apoyo de un sistema de gestión de aprendizaje. Praxis \& Saber, 7(14), 193-218. http://dx.doi.org/10.19053/22160159.5223

Chasi, N. S. (2020). Perfil profesional docente de educación general básica y el desempeño en el aula [Tesis de maestría, Universidad Técnica de Cotopaxi]. http://repositorio.utc.edu.ec/bitstream/27000/4501/1/PI-000727.pdf

Dávila, G., Leal, F., Comelin, A., Parra, M. y Varela, P. (2013). Conocimiento práctico de los profesores: sus características y contradicciones en el contexto universitario actual. Revista de La Educación Superior, XLII(166), 35-53. http://www.scielo.org.mx/pdf/resu/v42n166/v42n166a2.pdf

Doménech, J., Lope, S. y Mora, L. (2019). Qué proyectos STEM diseña y qué dificultades expresa el profesorado de secundaria sobre Aprendizaje Basado en Proyectos. Revista Eureka Sobre Enseñanza y Divulgación de Las Ciencias., 16(2), 1-16. https://doi.org/10.25267/rev_eureka_ensen_divulg_cienc.2019.v16.i2.2203

Espín, G. A. y Alpízar, R. (2020). Referentes bibliográficos sobre formación de competencias pe- dagógicas de los docentes universitarios. Revista Conrado, 16(74), 215-224. http://scielo.sld.cu/pdf/rc/v16n74/1990-8644-rc-16-74-215.pdf

Falcón, D., Sevil, J., Peñarrubia, C. y Abós, Á. (2021). Efecto de la combinación metodológica de aula invertida e instrucción entre pares en las calificaciones académicas de estudiantes universitarios de Ciencias de la Actividad Física y del Deporte. Retos, 41, 47-53. https://doi.org/10.47197/retos.v0i41.83984

Fernandez, J. (2016). Perfil profesional y perfil didáctico de los docentes del nivel secundaria de la I.E. Inca Garcilaso de la Vega comprendido en el ámbito del distrito de Huarmey, departamento de Ancash, año 2016 [Tesis, Universidad Católica Los Ángeles de Chimbote].

http://repositorio.uladech.edu.pe/handle/123456789/2159

Fernández, J. M. y Rodríguez, A. (2017). TIC y diversidad funcional: conocimiento del profesorado. European Journal of Investigation in Health, Psychology and 
Education, 7(3), 157-175. https://doi.org/10.30552/ejihpe.v7i3.204

Ferreiro, F. y Domínguez, D. (2020). Aprendizaje y práctica del diseño en contextos de diversidad: una aproximación basada en metodologías abiertas. Arte, Individuo y Sociedad, 33(1), 259-281. https://doi.org/10.5209/ARIS.68151

Flores, E., Escobar, N., Jara, P., Maureira, F., Gutiérrez, S. A., Cárdenas, S., Muñoz, M. y Díaz, V. (2021). Análisis del perfil de egreso de la carrera de pedagogía en educación física de Chile: un estudio cuantitativo. Retos, 39, 532-538. https://doi.org/10.47197/retos.v0i39.81379

García, J. y Pérez, J. E. (2018). Aprendizaje basado en proyectos: método para el diseño de actividades. $C E F, 10,37-63$. https://doi.org/10.51302/tce.2018.194

Gil, R. (2018). El uso del aprendizaje basado en problemas en la enseñanza universitaria: Análisis de las competencias adquiridas y su impacto. Revista Mexicana de Investigacion Educativa, 23(76), 73-93. http://www.scielo.org.mx/pdf/rmie/v23n76/1405-6666-rmie-23-76-73.pdf

Gómez, M. Á. y Quesada, V. (2017). Coevaluación o Evaluación Compartida en el Contexto Universitario: La Percepción del Alumnado de Primer Curso. Revista Iberoamericana de Evaluación Educativa, 10(2), 9-30. https://doi.org/10.15366/riee2017.10.2.001

González, R., Palomares, A., López, E. y Gento, S. (2019). Explorando el liderazgo pedagógico del docente: su dimensión formativa. Contextos Educativos, 24, 9-25. https://doi.org/10.18172/con.3936

Guerrero, C., Prieto, Y. y Noroña, J. (2017). La aplicación del aula invertida como propuesta metodológica en el aprendizaje de matemática. Espíritu Emprendedor TES, 2(1), 1-12. https://doi.org/10.33970/eetes.v2.n1.2018.33

Hinojo, F. J., Aznar, I., Romero, J. M.y Marín, J. A. (2019). Influencia del aula invertida en el rendimiento académico. Una revisión sistemática. Campus Virtuales, 8(1), 9-18.

https://redined.educacion.gob.es/xmlui/bitstream/handle/11162/184523/Art. 1.pdf? sequence $=1 \&$ is Allowed $=\mathrm{y}$

Luy, C. (2019). El Aprendizaje Basado en Problemas (ABP) en el desarrollo de la 
inteligencia emocional de estudiantes universitarios. Propósitos y Representaciones, 7(2), 353-368. https://doi.org/10.20511/pyr2019.v7n2.288

Marín, L. R., Suárez, J. M., López, Y. y Pelegrín, A. (2018). La clase encuentro en la educación superior: algunas consideraciones teóricas-metodológicas. ReHuSo: Revista de Ciencias Humanísticas y Sociales, 3(3), 88-100. https://www.redalyc.org/pdf/567/56752038009.pdf

Marín, V. y Cabero, J. (2019). Las redes sociales en educación: desde la innovación a la investigación educativa. RIED. Revista Iberoamericana de Educación a Distancia, 22(2), 25-33. https://dx.doi.org/10.5944/ried.22.2.24248

Martínez, M., Yániz, C. y Villardón, L. (2018). Autoevaluación y reflexión docente para la mejora de la competencia profesional del profesorado en la sociedad del conocimiento. RED. Revista de Educación a Distancia, 56, 1-31. http://dx.doi.org/10.6018/red/56/10

Muñoz, G. A., Rodríguez, P. y Luque, M. (2019). La formación inicial del profesorado de educación secundaria en España: perfil y motivaciones del futuro docente. Educación XX1, 22(1), 71-92. https://doi.org/10.5944/educXX1.20007

Orellana, R., Merellano, E. y Almonacid, A. (2018). Buen o buena docente de universidad: Perspectiva del personal directivo de carrera y de los mismos grupos docentes. Revista Electrónica Educare, 22(2), 1-27.

https://dx.doi.org/10.15359/ree.22-2.6

Pérez, L. (2018). El aprendizaje basado en problemas como estrategia didactica en educacion superior. Voces de La Educaicón, 3(6), 155-167. https://hal.archivesouvertes.fr/hal-02528555/document

Quijije, P. (2021). El docente universitario frente al perfil didáctico profesional. Reicomunicar, 4(7), 2-14. https://doi.org/10.46296/rc.v4i7.0021

Reyes, A. M., Cañon, M. J. y Olarte, F. A. (2018). Una propuesta de aula invertida en la asignatura de señales y sistemas de la Universidad Nacional de Colombia. Revista Educación En Ingeniería, 13(25), 82-87. https://doi.org/10.26507/rei.v13n25.877

Reyes, J. (2017). La planeación de clase; una tarea fundamental en el trabajo docente. Maestro y Sociedad, 14(1), 87-96. https://educrea.cl/wp- 
content/uploads/2018/10/DOC1-planeacion-tarea-fundamental.pdf

Rodríguez, J. y Alamilla, P. (2018). La complejidad del conocimiento profesional docente y la formación del conocimiento práctico del profesorado. Actualidades Investigativas En Educación, 18(2), 1-25.

https://doi.org/10.15517/aie.v18i2.33129

Sánchez, M. (2018). La evaluación del aprendizaje de los estudiantes : ¿es realmente tan complicada? Revista Digital Universitaria, 19(6), 1-18. https://doi.org/10.22201/codeic.16076079e.2018.v19n6.a1

Sánchez, P. P., López, M. M. y Alfonso, Y. (2018). La orientación educativa en la actividad pedagógica profesional del docente universitario. Revista Conrado, 14(65), 50-57. http://conrado.ucf.edu.cu/index.php/conrado

Segura, M. A. (2018). La función formativa de la evaluación en el trabajo escolar cotidiano. Revista Educación, 42(1), 1-20.

https://dx.doi.org/10.15517/revedu.v42i1.22743

Soto, E., Maldonado, G., Márquez, A. y Peña, N. (2021). Reconstruyendo el conocimiento práctico en confinamiento. Una experiencia de enseñanza en la formación inicial de docentes. RED. Revista de Educación a Distancia, 21(65), 139. https://doi.org/10.6018/red.450621

Suárez, M. (2021). Teoría y práctica de las metodologías participativas. Análisis de su utilización para la elaboración de agendas de desarrollo territorial en Uruguay. Prácticas y Discursos, 10(15), 1-20. http://dx.doi.org/10.30972/dpd.10154818

Tao, C. (2016). Contribución de la supervisión educativa al logro de los objetivos pedagógico-institucionales del Colegio Educativo Particular Peruano Chino Juan XXIII del distrito de San Miguel en el año 2015 [Tesis de maagíster, Universidad Nacional Mayor de San Marcos].

https://cybertesis.unmsm.edu.pe/handle/20.500.12672/4989

Toledo, P. y Sánchez, J. M. (2018). Aprendizaje Basado En Proyectos: Una Experiencia Universitaria. Profesorado, Revista de Currículum y Formación Del Profesorado, 22(2), 472-491. https://doi.org/10.30827/profesorado.v22i2.7733

Vásquez, S., Vásquez, S. A., Vásquez, C. A., Alania, R., Díaz, M. y del Águila, M. 
(2020). El perfil del docente investigador: hacia sus dimensiones y fortalecimiento The profile of the research teacher: towards its dimensions and its strengthening. Apuntes Universitarios, 10(4), 69-88. https://doi.org/10.17162/au.v10i4.492

Vera, J. Á., Bueno, G., Calderón, N. G. y Medina, F. L. (2018). Modelo de autoevaluación y heteroevaluación de la práctica docente en Escuelas Normales. Educ. Pesqui., São Paulo, 44, 1-19. http://dx.doi.org/10.1590/S1678$\underline{4634201844170360}$

Villarroel, V. A. y Bruna, D. V. (2017). Competencias Pedagógicas que Caracterizan a un Docente Universitario de Excelencia: Un Estudio de Caso que Incorpora la Perspectiva de Docentes y Estudiantes. Formacion Universitaria, 10(4), 75-96. https://doi.org/10.4067/S0718-50062017000400008

Vives, M. P. (2016). Modelos pedagógicos y reflexiones para las pedagogías del sur. Retos y Desafios de La Formación, 5(11), 40-55. https://revista.redipe.org/index.php/1/article/view/140

Zapata, M. (2016). Secuenciación de contenidos y objetos de aprendizaje Content sequencing and learning objects. RED. Revista de Educación a Distancia, 50(11), 1-29. https://doi.org/http://dx.doi.org/10.6018/red/50/11 